\title{
基于堵塞原理的变刚度软体机器人设计与试验*
}

\author{
徐丰羽 ${ }^{1}$ 蒋全胜 $^{2}$ 江丰友 $^{1}$ 申景金 ${ }^{1}$ 王兴松 $^{3}$ 蒋国平 $^{1}$ \\ (1. 南京邮电大学自动化学院 南京 210003; \\ 2. 苏州科技大学机械工程学院 苏州 215009; \\ 3. 东南大学机械工程学院 南京 211189)
}

\begin{abstract}
摘要: 软体机器人运动时具有高柔性, 执行任务时又能展示出强刚度, 在军事侦察、灾难救援等复杂环境探索与检测方面具 有重要的应用价值。结合主动驱动的网络气动结构与被动驱动的堵塞机构的优势, 提出实时变刚度的软体驱动器, 研究其变 刚度机理和动态建模方法。首先, 提出了气动-堵塞机构耦合的软体驱动器模型; 其次, 利用赫兹接触模型, 建立机器人运动 数学模型, 从理论上研究其变刚度形成机理; 再次, 利用有限元对气动驱动结构进行分析, 研究空腔内压强、形状和大小对 软体机器人弯曲角度的影响, 并进行了优化; 最后, 制作了变刚度软体机械臂样机, 验证了软体驱动器的变刚度性能与运动 性能。该研究有望为变刚度软体机器人设计与刚度调控提供新的理论和技术支持。
\end{abstract}

关键词: 变刚度; 颗粒堵塞; 软体驱动器; 打印; 试验研究

中图分类号: TP242

\section{Design and Testing of a Soft Robot with Variable Stiffness Based on Jamming Principles}

\begin{abstract}
XU Fengyu $^{1}$ JIANG Quansheng ${ }^{2} \quad$ JIANG Fengyou ${ }^{1}$ SHEN Jingjin ${ }^{1}$ WANG Xingsong $^{3}$ JIANG Guoping ${ }^{1}$

(1. College of Automation, Nanjing University of Posts and Telecommunications, Nanjing 210003;

2. College of Mechanical Engineering, Suzhou University of Science and Technology, Suzhou 215009;

3. College of Mechanical Engineering, Southeast University, Nanjing 211189)

Abstract: A soft robot has high flexibility when performing tasks with moving and high stiffness, so it has important application in exploring and detecting complex environments. Based on the advantages of driving Pneu-Net and driven jamming mechanism, a soft robot with real-time variable stiffness and dynamic modeling methods is proposed. Firstly, the model of the soft actuator coupling pneumatic structure and jamming mechanism is established. Secondly, by using Hertzian contact model, a mathematical model of robot motion and theoretically investigated formation mechanisms of variable stiffness is built. Moreover, the pneumatically-driven structure is analyzed by using the finite element as well as the influences of pressure in cavities, considering shape and size of cavities on the bending angle of the soft robot are studied. Based on this, the pneumatically-driven structure is optimized. Finally, A prototype and testing of the soft robot arm with variable stiffness is implemented, which verified the variable stiffness and motion performances of the soft actuator. This study provides new theoretical and technical support for the design and stiffness control of a soft robot with variable stiffness.
\end{abstract}

Key words: variable stiffness; particle jamming; soft robot; three-dimensional printing; experimental research

\section{0 前言}

基于堵塞原理的驱动器是变刚度结构的典型代

* 国家自然科学基金资助项目(51775284)、江苏省重点研发计划(社会发 展, BE2018734)、江苏省第十五批 “六大人才高峰” 高层次人才项目 (JY-081)和江苏省自然科学基金面上项目(BK20201379)资助项目。 20200405 收到初稿, 20200820 收到修改稿
表，能实现流体状态和固体状态之间相互转化。目 前有 2 种堵塞驱动方法: 薄膜内充满颗粒材料和薄 膜内充满薄片状材料。这两种方法原理相同，均是 利用施加, 增加弹性薄膜内材料的剪应力; 不同点 在于, 颗粒堵塞在流体状态下呈高变形性, 对外界 适形能力强; 薄片堵塞变形能力略差, 但可提供的 刚度变化范围较大。

BROWN 教授 ${ }^{[1]}$ 利用堵塞原理设计了一种万能 
手爪, 可抓持不规则表面的物体, 柔性夹持器可根 据物体的形状在其周围 “流动”, 适应其形状, 施加 真空作用后, 颗粒迅速收缩抓持物体。HOU 等 ${ }^{[2]}$ 利 用负压和颗粒流实现变刚度, 提出了一种通用变刚 度手爪, 具有更高的负载能力。ZHU 等 ${ }^{[3]}$ 应用打印 多种材料设计了变刚度软体机器人夹持器, 包含两 个软性手指。针对传统机构弯曲角度较小等问题, $\mathrm{LI}$ 等 ${ }^{[4-5]}$ 提出了分布式颗粒堵塞机构, 在保持转动刚 度的同时, 大大提高了颗粒阻塞夹持器的弯曲能力。 Versaball ${ }^{[6]}$ 是为数不多的商业化万能夹持器之一, 对 于堵塞机构有较大参考意义。

作为半主动驱动方法, 堵塞机构经常与其他驱 动方式联合。

（1）堵塞机构可与特殊机械结构结合形成变刚 度机构：WEI 等 ${ }^{[7]}$ 将多个串联的球形关节与颗粒堵 塞机构集成, 提出了可调刚度机械臂, 相比没有颗 粒堵塞的机械臂刚度提高了 13 倍。WEI 等 ${ }^{[8]}$ 提出的 这种可变刚度抓手具有可控制的自由度的特征, 从 而扩展了它们的环境适应性和操纵能力。WU 等 ${ }^{[9]}$ 将刚性的内骨骼嵌入软指的内腔, 提出了一种新型 的具有关节内骨骼结构的可变刚度抓手。

(2) 堵塞机构可与绳索驱动集成: KIM 等[10] 将 $\mathrm{NiTi}$ 绳驱动技术与分层堵塞技术集成, 设计了仿 蛇机器人, 获得了与微创手术相融合的机器人特性。 CHENG 等 ${ }^{[11]}$ 将绳索驱动与颗粒堵塞原理相结合设 计了模块化机械手臂, 实现了局部刚度的控制。LIN 等 ${ }^{[12]}$ 提出了一种变刚度驱动器, 将气动致动、真空 阻塞和肌腱驱动集成在一起, 可应用多种方式调解 刚度, 实现 10 倍以上变刚度。ZHANG 等 ${ }^{[13]}$ 提出一 种采用绳索驱动的拮抗式变刚度柔性机器人关节, 能够根据任务需要, 实时调节关节刚度。

(3) 堵塞机构还可与流体驱动结合: WALL 等 ${ }^{[14]}$ 将堵塞机构与气体驱动器相结合, 提出变刚度手指。 WEI 等 ${ }^{[15]}$ 提出了可自适应抓取的变刚度软体夹持 器, 对物体适形后, 通过真空作用可迅速固化使手 指保持既有形态, 刚度提高 10 倍以上。BROWN 等 ${ }^{[16]}$ 设计了一款新型软体移动机器人, 在流体状态 球形机器人表面布置多个堵塞单元, 通过堵塞部分 单元格, 实现滚动运动。其他较有创新的堵塞机构 包括: JIANG 等 ${ }^{[17]}$ 提出了一种基于差压驱动的新型 颗粒阻塞机构。WANG 等 ${ }^{[18]}$ 提出了一种新型层阻塞 变刚度技术。YAO 等 ${ }^{[19]}$ 设计了一种气动式可变刚度 的软体机械臂模块, 实现了软体机械臂的模块化。 WANG 等 ${ }^{[20]}$ 对软体机器人目前的发展现状进行了 系统的总结, 并提出可变刚度研究方向可能是未来
软体机器人研究新的突破点。

综上所述，堵塞机构多应用小尺寸颗粒材料， 由于小颗粒易发生变形和重新排列, 可能导致不同 运动形态和变刚度失效, 降低了机构重复定位精度, 甚至定位错误。科研人员目前多试图从试验的角度 去分析影响堵塞机构的主要因素 ${ }^{[21-23]}$, 包括: 分析 颗粒数量、形状、尺寸和弹性薄膜的机械特性对刚 度的影响。

变刚度的主动驱动方式和半主动驱动方式各有 优缺点, 将二者结合可形成功能强大软体机器人。 本文提出了一种基于大、小颗粒组合的堵塞机构, 提出了一种气动-堵塞机构耦合的变刚度软体驱动 器; 利用赫兹接触模型, 建立变刚度结构数学模型, 分析其变刚度形成机理; 应用 Abaqus 有限元软件对 气动驱动结构进行分析, 研究空腔内压强、空腔形 状和空腔大小对弯曲角度的影响; 制作了软体机器 人样机, 搭建刚度测试平台, 验证了变刚度软体机 器人的弯曲性能和稳定性。

\section{1 变刚度软体机器人模型}

所提出的变刚度软体机器人, 主要采用模块化 设计思想, 将复杂的软体机器人结构分解为单个模 块的变刚度软体驱动器相连而成。软体驱动器由三 层组成(图 1), 最内层为堵塞变刚度结构, 底部与颗 粒堵塞真空管相连; 中间层为气动驱动结构, 布置 在堵塞结构周围, 气动驱动结构内设有 3 个成 $120^{\circ}$ 的空腔通道, 空腔通道底部与气动加压管相连; 最 外层为硅胶-纤维复合结构, 用来限制气动结构过度 膨胀。

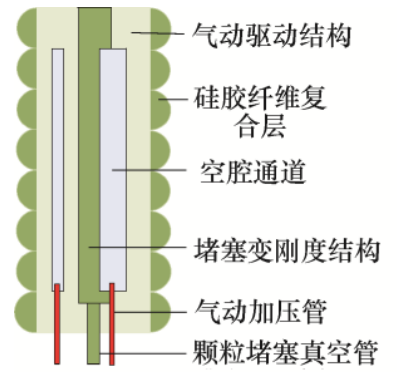

(a) 单元驱动器正视图

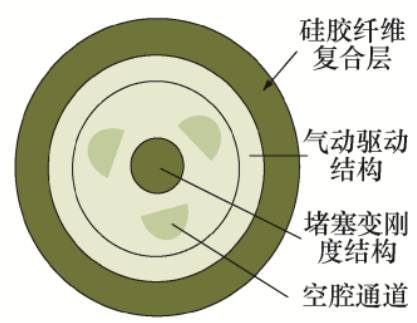

(b) 单元驱动器俯视图
图 1 单元驱动器结构

\section{1 堵塞变刚度结构}

堵塞变刚度结构形状为圆柱体, 结构见图 2a。 结构底部通过颗粒堵塞真空管与外界真空泵相连, 外部由薄膜包裹而成, 薄膜内部的多个菱形骨架通 
过柔性绳索连接, 大球颗粒依靠收紧弹簧嵌切在 2 个菱形骨架之间，空隙填充微小颗粒。

其工作原理为: 加载真空压力后, 膜内的小颗 粒物质从流动状态转变为固体状态; 在固体状态下, 密封薄膜将大球颗粒和菱形骨架密封成一体, 大球 颗粒和关节骨架间填充有细小颗粒(图 2b), 当机构 运动时, 大球颗粒始终嵌切于关节骨架之间, 所有 颗粒均不会随机散落, 通过控制膜内真空度来调整 机器人刚度。该堵塞机构, 克服了小颗粒堵塞机构 在运动过程中颗粒重组导致位置不确定甚至定位错 误的缺点。

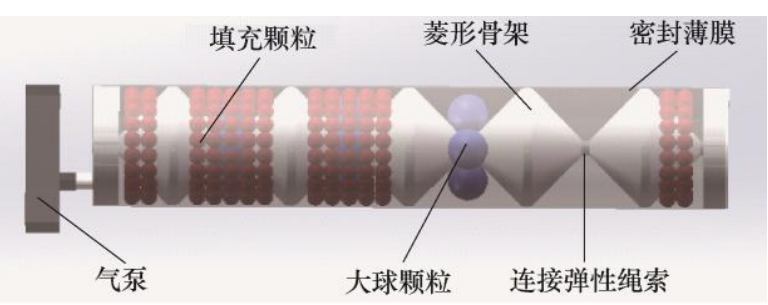

(a) 初始自由状态

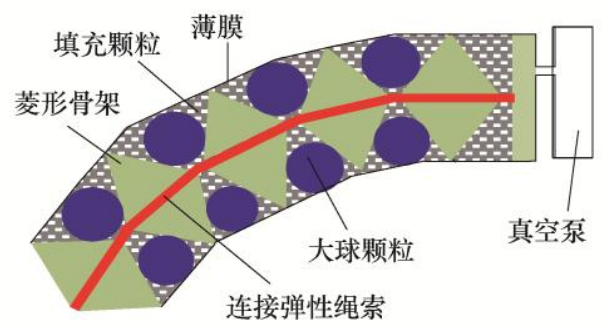

(b) 弯曲伸长状态

图 2 大小颗粒组合式堵塞变刚度机构

\section{2 气动驱动结构}

气动驱动结构由弹性材料制成, 在其圆周方向 上设置了 3 个空腔通道。1 个或 2 个空腔加载时, 产生弯曲; 当 3 个外部空腔同时充气, 驱动器沿轴 向伸长; 控制不同空腔内压力可调整弯曲的角度和 方向, 从而实现万向弯曲运动。

\section{3 硅胶纤维复合层}

当空腔气动加载时, 外壁可能会过度膨胀, 发 生气球效应。硅胶纤维复合层是由多个硅胶纤维构 成, 外部呈环状, 由弹性模量较大的材料制成。将 多个单环状纤维嵌入驱动器表面, 仅限制了驱动器 的径向过度膨胀, 对其轴向拉伸、压缩和弯曲影响 较小。同时, 这种纤维复合结构可提高抓取物体时 的 “粘滞力”, 能提供较大的末端力, 弯曲效率高。

\section{2 变刚度软体机器人数学建模}

机构的刚度主要由小颗粒, 大球和菱形骨架三 者间摩擦力所决定。自由状态下, 颗粒可自由运动,
当施加致动气压时, 膜内外压力差将小颗粒、菱形 骨架和大球挤压在一起, 形成锁紧力, 不仅限制了 小颗粒的移动, 同时也固定了菱形骨架和大球的位 置, 获得较高刚度以抵抗外力。

\section{1 颗粒间锁紧力建模}

为了分析堵塞颗粒对刚度的影响, 当施加真空 负压时, 假定膜内的所有颗粒都为刚性, 且被限制 为一个整体。图 $3 \mathrm{a}$ 为堵塞机构的单个模块的结构 示意图, 当受外力矩 $M$ 时, 简化受力分析见图 3b, 图 3c 为模块的剖面图, 将薄膜内的大球和颗粒分为 上、下两层, 下层颗粒块的锁紧力抵抗外力矩 $M$ 。 端部模块的锁紧力分析见图 $3 \mathrm{~d}$, 当末端受外力 $F_{\mathrm{E}}$ 时, 两个颗粒层沿水平方向发生相对滑动, 对下层 颗粒块(图 $3 \mathrm{~d}$ 锁紧区域)受力分析, 锁紧力 $F_{\mathrm{L}}$ 为

$$
F_{\mathrm{L}}=2 f_{\mathrm{U}}+f_{\mathrm{P}}+f_{\mathrm{L}}
$$

式中, 是 $f_{\mathrm{L}}$ 颗粒块与接触区域 $A_{1}$ (图 3b) 的摩擦力, $f_{\mathrm{P}}$ 是颗粒块在锁紧区域 $(3 \mathrm{~d})$ 上的静态力, $f_{\mathrm{U}}$ 是下层颗 粒块与大球水平方向上的摩擦力。

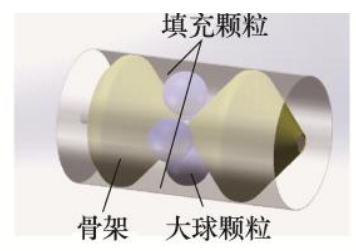

(a) 末端模块

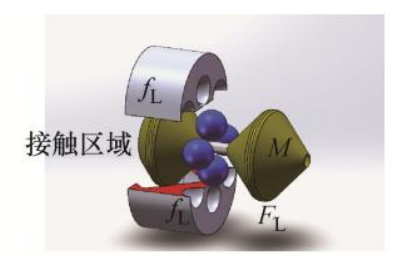

(b) 受负载影响末端模块

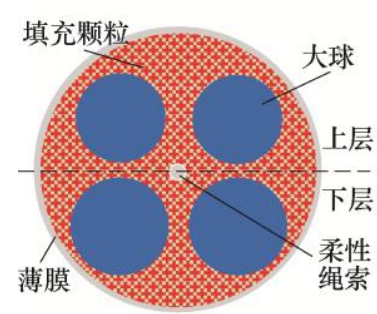

(c) 末端模块剖面图

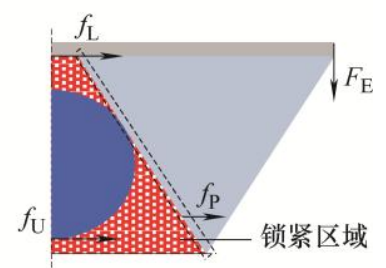

(d) 末端模块底层分析
图 3 锁紧力示意图

颗粒层在锁紧区域上的静态力 $f_{\mathrm{P}}$, 可通过流体 加压模型来估算，密闭空间内流体的压强能够大小 不变的传向各个方向, 见图 $4 \mathrm{a}$, 可得

$$
P_{1}=P_{1}=P_{1}=P_{1}=\frac{F_{\text {ext }}}{A_{2}}
$$

所以, 施加在区域 $A_{1}$ 上的力 $F_{A 1}$ 为

$$
F_{A 1}=\frac{F_{\text {ext }}}{A_{2}} A_{1}
$$

将小颗粒等效成流体，薄膜等效为一个密闭空间， 其内部被小颗粒填满, 构成如图 $4 \mathrm{~b}$ 所示的静态流体 模型, 施加在每一面的力可以通过式(3)计算。颗粒 块作用在锁紧区域 $A_{3}$ 上水平方向上的静态力 $f_{\mathrm{P}}$ 由颗 
粒块与菱形骨架间的压力提供

$$
f_{\mathrm{P}}=F_{\mathrm{N} 2} \sin \alpha=p^{\prime} A_{3} \sin \alpha=\pi R_{1}^{2} p^{\prime} \sin \alpha
$$

式中, $F_{\mathrm{N} 2}$ 为颗粒块对菱形骨架的正压力, $R_{1}$ 为薄 膜管半径, $\alpha$ 为菱形骨架顶角, $P^{\prime}$ 为颗粒块施加给 菱形骨架和大球的等效压强。则颗粒块在与菱形骨 架接触区域 $A_{1}$ 上的摩擦力 $f_{\mathrm{L}}$ 为

$$
f_{\mathrm{L}}=\mu_{1} P^{\prime} A_{1}=\pi R_{1}^{2} P^{\prime} \mu_{1}
$$

式中, $\mu_{1}$ 为颗粒块与骨架之间的静摩擦因数, $A_{1}$ 为 颗粒块与菱形骨架接触区域的等效面积。大球与颗 粒块之间在水平方向的摩擦力 $f_{\mathrm{U}}$ 为

$$
f_{\mathrm{U}}=\mu_{2} F_{\mathrm{N} 1}=\mu_{2} P^{\prime} A_{2}=\pi R_{2}^{2} P^{\prime} \mu_{2}
$$

式中, $F_{\mathrm{N} 1}$ 为颗粒块对大球的正压力, $R_{2}$ 为大球半 径, $\mu_{2}$ 为颗粒与大球之间的静摩擦因数, $A_{2}$ 为颗粒 块与大球接触的等效面积。将式(4) (6)代入式(1), 则锁紧力 $F_{\mathrm{L}}$ 为

$$
\begin{gathered}
F_{\mathrm{L}}=\pi R_{1}^{2} P^{\prime} \sin \alpha+2 \pi R_{2}^{2} P^{\prime} \mu_{2}+\pi R_{1}^{2} P^{\prime} \mu_{1}= \\
\pi \mathrm{P}^{\prime}\left(R_{1}^{2} \sin \alpha+2 R_{2}^{2} \mu_{2}+R_{1}^{2} \mu_{1}\right)
\end{gathered}
$$

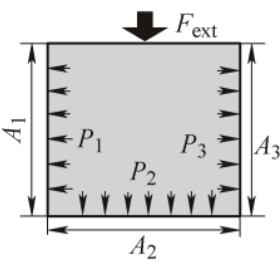

(a) 流体加压模型

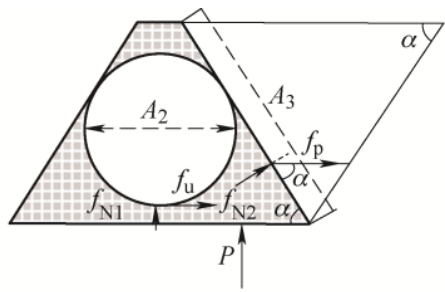

(b) 颗粒堵塞机构力分析模型
图 4 颗粒液体模型

\section{2 赫兹接触模型}

根据两圆球赫兹接触模型(图 5a), 则两球体相 接触后, 其中心相对变形位移和应力分别为

$$
\begin{gathered}
\delta^{3}=\frac{9}{16} \frac{R_{\mathrm{a}}+R_{\mathrm{b}}}{R_{\mathrm{a}} R_{\mathrm{b}}}\left(\frac{1-v_{\mathrm{a}}^{2}}{E_{\mathrm{a}}}+\frac{1-v_{\mathrm{b}}^{2}}{E_{\mathrm{b}}}\right)^{-2} P \\
P^{\prime 3}=\frac{6}{\pi^{3}}\left(\frac{R_{\mathrm{a}}+R_{\mathrm{b}}}{R_{\mathrm{a}} R_{\mathrm{b}}}\right)^{2}\left(\frac{1-v_{\mathrm{a}}^{2}}{E_{\mathrm{a}}}+\frac{1-v_{\mathrm{b}}^{2}}{E_{\mathrm{b}}}\right)^{-2} P
\end{gathered}
$$

式中, $R_{\mathrm{a}}$ 为 $\mathrm{a}$ 球半径, $R_{\mathrm{b}}$ 为 $\mathrm{b}$ 球半径, $v_{\mathrm{a}}$ 为 $\mathrm{a}$ 球泊 松比, $v_{\mathrm{b}}$ 为 $\mathrm{b}$ 球泊松比, $E_{\mathrm{a}}$ 为 $\mathrm{a}$ 球弹性模量, $E_{\mathrm{b}}$ 为 $\mathrm{b}$ 球弹性模量。自然状态下，小颗粒半径 $R_{3}$ 远小于 薄膜管半径 $R_{1}$, 单个颗粒正对着的薄膜区域面积较 小, 可近似的看成是平面。利用赫兹接触理论对薄 膜和颗粒块进行分析, 可建立如图 5b 所示的球体平面赫兹接触模型, 令公式(8)、(9)中 $R_{\mathrm{a}}=\infty, R_{\mathrm{b}}=R_{3}$ 将薄膜管和小颗粒的参数代入，得

$$
\delta^{3}=\frac{9}{16 R_{3}}\left(\frac{1-v_{1}^{2}}{E_{1}}+\frac{1-v_{2}^{2}}{E_{2}}\right)^{-2} P
$$

$$
P^{\prime 3}=\frac{6}{\pi^{3} R_{3}^{2}}\left(\frac{1-v_{1}^{2}}{E_{1}}+\frac{1-v_{2}^{2}}{E_{2}}\right)^{-2} P
$$

式中, $R_{3}$ 为填充颗粒半径, $v_{1}$ 为菱形骨架的泊松比, $v_{3}$ 为小颗粒泊松比, $E_{1}$ 为菱形骨架弹性模量, $E_{3}$ 为 填充颗粒弹性模量。

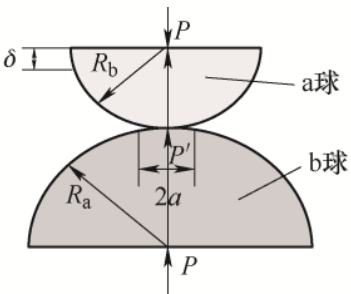

(a) 两圆球接触赫兹模型

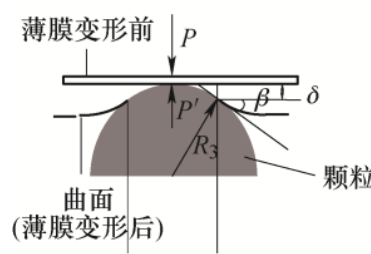

(b) 颗粒与平面赫兹接触模型

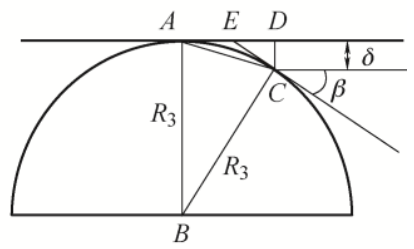

(c) 简化模型
图 5 颗粒与平面接触模型

颗粒与平面接触赫兹简化模型见图 $5 \mathrm{c}$ ，由等 腰 $\triangle A B C 、 \triangle A E C$ 和直角 $\triangle A D C 、 \triangle E D C$ 的性质, 可得

$$
\begin{gathered}
A C=\sqrt{2 R_{3}^{2}-2 R_{3}^{2} \cos \angle A B C} \\
A C=\frac{\delta}{\sin \angle D A C} \\
\angle A B C=2 \angle D A C=\angle \beta
\end{gathered}
$$

联立式(12)、(13)、(14), 得薄膜与小球颗粒接触变 形后其变形角 $\beta$ 为

$$
\begin{gathered}
\cos \beta=\frac{2 R_{3}-\sqrt{2} \delta}{2 R_{3}}= \\
1-\left[\frac{9}{32 \sqrt{2} R_{3}^{4}}\left(\frac{1-\mu_{1}^{2}}{E_{1}}+\frac{1-\mu_{2}^{2}}{E_{2}}\right) p\right]^{\frac{1}{3}}
\end{gathered}
$$

机构末端的锁紧力 $F_{\mathrm{L}}$ 为

$$
\begin{gathered}
F_{\mathrm{L}}=\left[\frac{6}{\pi^{3} R_{3}^{2}}\left(\frac{1-v_{1}^{2}}{E_{1}}+\frac{1-v_{2}^{2}}{E_{2}}\right)^{-2}\right]^{\frac{1}{3}} \times \\
\left(R_{1}^{2} \mu_{1}+R_{1}^{2} \sin \alpha+2 R_{2}^{2} \mu_{2}\right) P^{\frac{1}{3}}
\end{gathered}
$$

机构的锁紧力矩 $M_{\mathrm{L}}$ 为

$$
\begin{aligned}
M_{\mathrm{L}}= & F_{\mathrm{L}} \cdot d=\left[\frac{6}{\pi^{3} R_{3}^{2}}\left(\frac{1-v_{1}^{2}}{E_{1}}+\frac{1-v_{2}^{2}}{E_{2}}\right)^{-2}\right]^{\frac{1}{3}} \times \\
& \left(R_{1}^{2} \mu_{1}+R_{1}^{2} \sin \alpha+2 R_{2}^{2} \mu_{2}\right) P^{\frac{1}{3}} d
\end{aligned}
$$


式中, $r$ 为薄膜管半径, 取极限真空度 $P_{\mathrm{m}}=101 \mathrm{kPa}$, 并代入式(17)中, 则最大锁紧力矩 $M_{\mathrm{Lm}}=304.5 \mathrm{~N} / \mathrm{mm}$ 。

根据经典线弹性理论, GENT 提出弹性模量值 $E$ 与肖氏硬度值 $S$ 的关系为 ${ }^{[24]}$

$$
E=\frac{0.0981(56+7.62336 S)}{0.137505(254-2.54 S)}
$$

式中, $E$ 为弹性模量, $S$ 是以 ASTM D2240 标准的 硬度计示数。联合式(17)、(18), 可得小颗粒硬度与 锁紧力矩的关系

$$
\begin{gathered}
M_{\mathrm{L}}=\left[\frac{6}{\pi^{3}}\left(\frac{0.137505\left(254-2.54 S_{1}\right)}{0.0981\left(56+7.62336 S_{1}\right)}+\frac{1-v_{2}^{2}}{E_{2}}\right)^{-2}\right]^{\frac{1}{3}} \times \\
\left(R_{1}^{2} \mu_{1}+R_{1}^{2} \sin \alpha+2 R_{2}^{2} \mu_{2}\right) P^{\frac{1}{3}} d
\end{gathered}
$$

\section{3 变刚度特性仿真}

将软体机器人的参数设置为: 菱形骨架半径, $R_{1}=14 \mathrm{~mm}$ 大球颗粒半径 $R_{2}=6.5 \mathrm{~mm}$, 填充颗粒半径 $R_{3}=3 \mathrm{~mm}$, 根据式(15), 得薄膜变形角 $\beta$ 与真空压力 $P$ 之间的关系(图 6), 由图可得, 真空压力 $P$ 越大, 薄膜变形角 $\beta$ 也越大。同时, 随着压力逐渐增大, 压 力增量相同时, 薄膜变形角的增量不断减小。

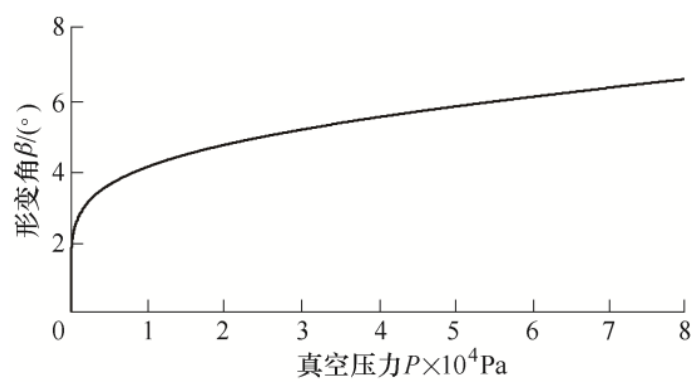

图 6 薄膜变形角 $\beta$ 与真空压力 $P$ 关系曲线

将参数设置为: $R_{1}=14 \mathrm{~mm}, R_{2}=6.5 \mathrm{~mm}$, $R_{3}=3 \mathrm{~mm}$, 薄膜管半径 $r=16 \mathrm{~mm}$ 。根据公式(16), 得到合力矩 $M$ 和真空压力 $P$ 之间的关系(图 7)。从 图 7 可见, 真空压力 $P$ 越大, 可承受外力矩 $M$ 越大, 故可通过调整真空压力来控制机构刚度。

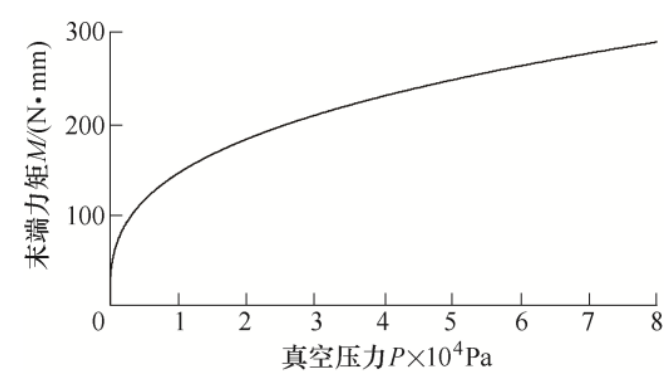

图 7 末端力矩 $M$ 与真空压力 $P$ 的关系曲线

将机构的参数设置为: $R_{1}=14 \mathrm{~mm}$, $R_{2}=6.5 \mathrm{~mm}, P=80 \mathrm{kPa}, r=16 \mathrm{~mm}$, 并将 $R_{3}$ 范围
设置在 $2 \sim 6 \mathrm{~mm}$ 之间。根据式(17), 得外力矩 $M$ 与小颗粒半径 $R_{3}$ 之间的关系(图 $8 \mathrm{a}$ )。从图 8 可知, 小颗粒半径 $R_{3}$ 越大，末端力矩 $M$ 越小。且随着小 颗粒尺寸的减小，相同尺寸减量下，末端力矩的减 量越来越小。各参数对比如表 1 所示。

\section{表 1 变刚度仿真参数表}

\begin{tabular}{cccccc}
\hline $\begin{array}{c}\text { 曲线图参数 } \\
\text { 设置 }\end{array}$ & $\begin{array}{c}\text { 菱形骨架 } \\
R_{1} / \mathrm{mm}\end{array}$ & $\begin{array}{c}\text { 大球半径 } \\
R_{2} / \mathrm{mm}\end{array}$ & $\begin{array}{c}\text { 小颗粒半 } \\
\text { 径 } R_{3} / \mathrm{mm}\end{array}$ & $\begin{array}{c}\text { 薄膜管半 } \\
\text { 径 } r / \mathrm{mm}\end{array}$ & $\begin{array}{c}\text { 真空压力 } \\
P / \mathrm{kPa}\end{array}$ \\
\hline$\beta$ 与 $P$ & 14 & 6.5 & 3 & 16 & $0 \sim 80$ \\
$M$ 与 $P$ & 14 & 6.5 & 3 & 16 & $0 \sim 80$ \\
$M$ 与 $R_{3}$ & 14 & 6.5 & $2 \sim 6$ & 16 & 80 \\
$M$ 与 $R_{1}$ & $10 \sim 15$ & 6.5 & 3 & 16 & 80 \\
$M$ 与 $R_{2}$ & 14 & $3 \sim 7$ & 3 & 16 & 80 \\
$M$ 与 $S_{1}$ & 14 & 6.5 & 3 & 16 & 80 \\
\hline
\end{tabular}

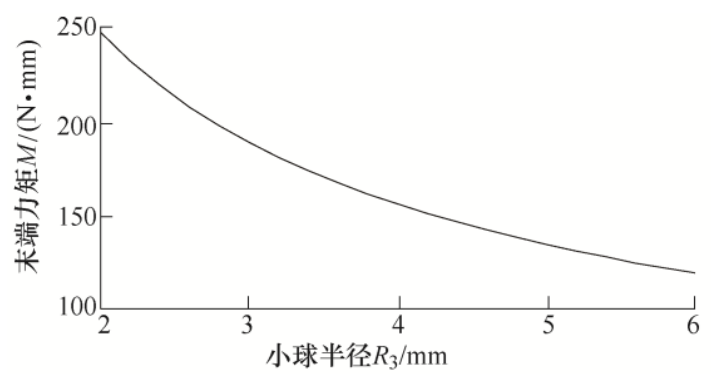

(a) 末端力矩 $M$ 与小颗粒半径 $R_{3}$ 的关系曲线

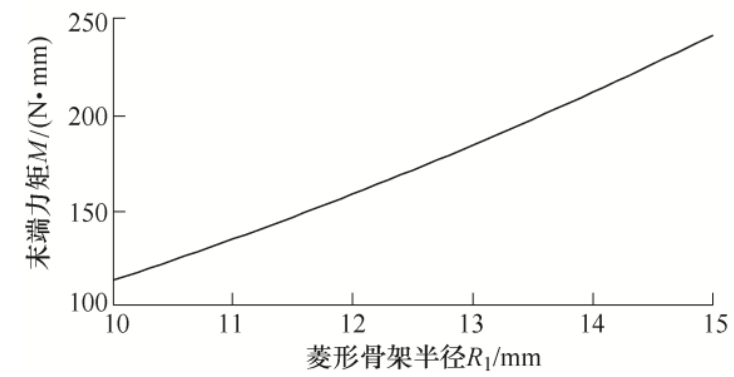

(b) 末端力矩 $M$ 与菱形骨架半径 $R_{1}$ 的曲线关系

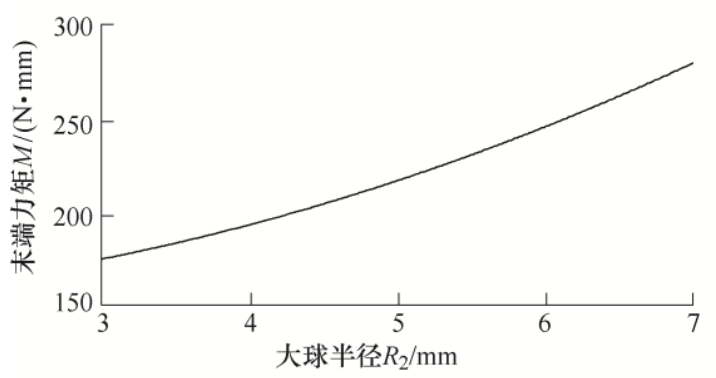

(c) 末端力矩 $M$ 与大球半径大小 $R_{2}$ 的对应关系

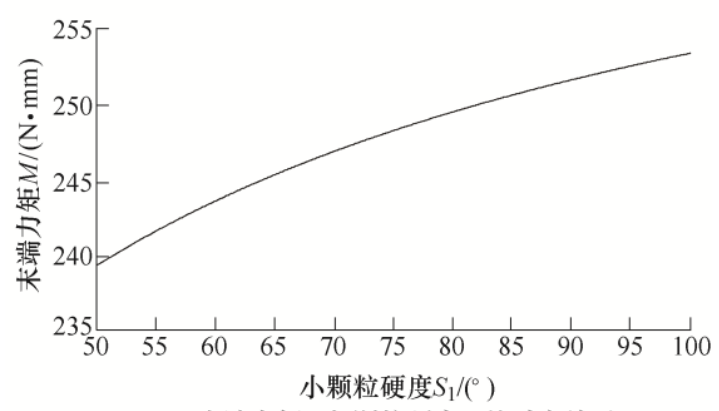

(d) 末端力矩 $M$ 与颗粒硬度 $S_{1}$ 的对应关系

图 8 机构末端承受力矩仿真结果 
将机构的参数设置为: $R_{2}=6.5 \mathrm{~mm}, R_{3}=3 \mathrm{~mm}$, $r=16 \mathrm{~mm}, P=80 \mathrm{kPa}$, 并将菱形骨架半径 $\mathrm{R}_{1}$ 范围 设置在 $10 \sim 15 \mathrm{~mm}$ 之间。根据式(17), 得末端力矩 $M$ 与菱形骨架 $R_{1}$ 之间的关系(图 $8 \mathrm{~b}$ ), 可得, 菱形骨 架的半径 $R$ 越大，末端力矩 $M$ 越大。

将机构的参数设置为: $R_{1}=14 \mathrm{~mm}, R_{3}=3 \mathrm{~mm}$ $r=16 \mathrm{~mm}, P=80 \mathrm{kPa}$, 并将大球颗粒半径 $\mathrm{R}_{2}$ 设置 为 3 7 mm 之间。根据式(17), 可得末端外力矩 $M$ 与大球半径尺寸 $R_{2}$ 之间的关系(图 8c)。可见大球半 径尺寸越大, 机构末端的合力矩越大。且随着大球 半径的增大, 相同尺寸增量下, 末端力矩的增量越 来越大。

将机构的参数设置为: $R_{1}=14 \mathrm{~mm}$, $R_{2}=6.5 \mathrm{~mm}, R_{3}=3 \mathrm{~mm}$ 。根据式(17), 得末端力 矩 $M$ 与颗粒硬度 $S_{1}$ 之间的关系(图 8d)。从图 8 中可见, 小颗粒硬度 $S_{1}$ 越大, 机构的末端力矩 $M$ 越大。且随着小颗粒硬度的增大, 相同增量下, 末端力矩的增量越来越小。

\section{3 机器人样机制作}

为验证机器人可变刚度能力, 制作了机器人样 机, 搭建了机器人测试平台, 完成了机器人变刚度 试验。

\section{1 变刚度软体驱动器制备}

根据第 2 节提出的结构模型, 设计了机器人的 主要组件见图 9, 堵头和菱形骨架均留有半径 $4 \mathrm{~mm}$ 的圆孔, 供柔性绳索穿过, 在堵头模型中, 上通孔 供柔性绳索穿过, 下通孔与气管相连, 供气流进出。 这 3 个组件经过 SolidWorks 设计后, 应用 PLA 材料 打印制造。

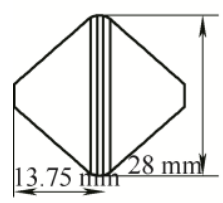

(a) 骨架模型图

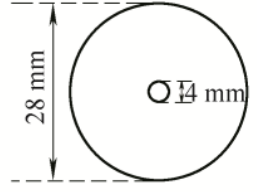

(b) 堵头模型

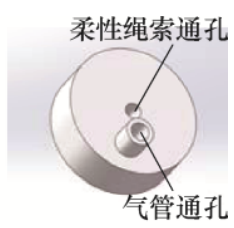

(c) 通气堵头
图 9 变刚度软体驱动器组件模型

柔性绳索采用 $2.8 \mathrm{~mm}$ 的弹力皮筋, 薄膜采用厚 度为 $0.24 \mathrm{~mm}$ 的 $\mathrm{PE}$ 材料制成, 填充颗粒选用半径 为 $3 \mathrm{~mm}$ 的刚玉材质颗粒。

具体装配流程: 先将柔性绳索穿过堵头和等间 距的放置菱形骨架的中心通孔, 接着应用胶水进行 胶粘固定。同时, 将气管与通气堵头的凸槽相连接 (图 10a)。再将该组件的菱形骨架逐个装入薄膜中,
并填充颗粒。最后, 使用硅酮胶对两端密封, 堵塞 机构样机见图 10b。

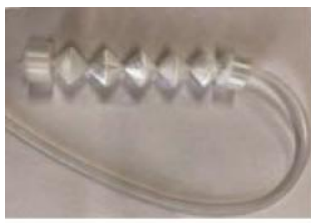

(a) 菱形骨架与堵头装配图

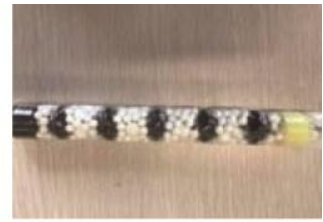

(b) 变刚度机构
图 10 变刚度结构实物效果图

\section{2 气动驱动结构制备}

气动驱动结构选用壁厚 $2 \mathrm{~mm}$ 的半圆形空腔, 为了便于模具拆除, 采用目前主流的分离式模具设 计方法, 利用 3D 打印机制造模具, 再按照图 11 流 程进行装配: (1) 将底座和堵塞机构模具进行组装 (图 11a); (2) 将 3 个空腔模具的气孔端插入底座上 预留的空隙(图 11b); (3) 将 2 个外环套筒, 分别套 在模具外侧并组装(图 11c); (4) 将封口膜缠绕在 外壳和底座的表面, 用皮筋䈐紧; (5) 将调制好的 ECOFLEX-0030硅胶注入模具中; (6) 整体加热待 硅胶凝固后, 移除底座 1 和空腔模具, 翻转柔性 体, 将底座 2 模具与套筒进行组装(图 11e), 外部 套上封口膜; (7) 继续将调制好的 Dragon Skin 硅 胶注入, 充满底座 2 模具间隙, 并与柔性体充分 接触; (8) 整体再加热, 凝固后移除全部模具; (9) 在空腔细孔处插入硅胶软管, 应用 703 硅胶粘 接和密封(图 11f)。

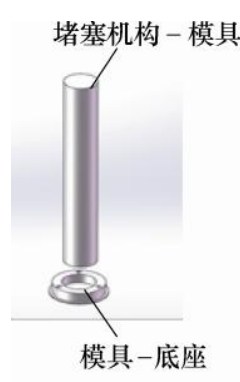

(a) 底座模具

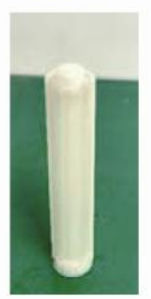

(d) 整体模具

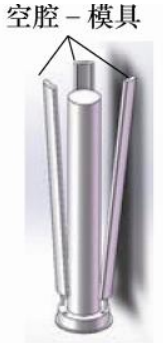

(b) 空腔装配

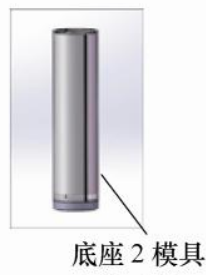

(e) 浇注顶盖

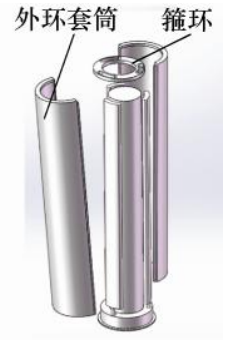

(c) 外环套筒与膂 环装配

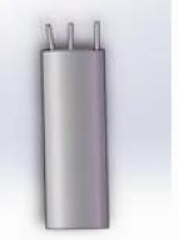

(f) 接入气管
图 11 软体驱动器制作流程

\section{3 硅胶纤维复合层制备}

采用同样方法, 设计的硅胶纤维复合层模具见 
图 12a, 经 3D 打印后, 与底座进行组装(图 12b), 再将气动驱动结构放置在内, 将配置好的液态硅胶 注入模具, 制作纤维复合层, 成型后移除模具。液 态硅胶采用弹性模量较大的 Dragon skin-10 硅胶配 制而成, 该材料的弹性模量和硬度远高于 Ecoflex-0030。

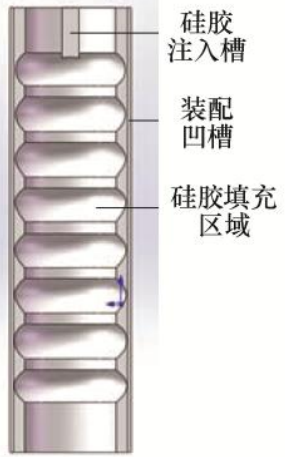

(a) 纤维复合层模具

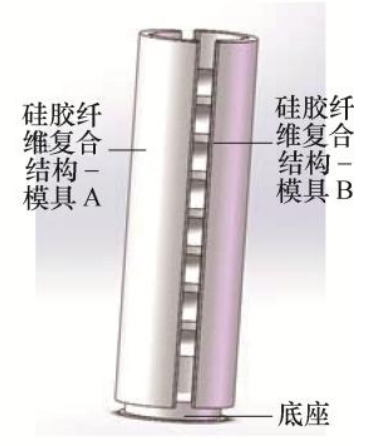

(b) 模具装配图

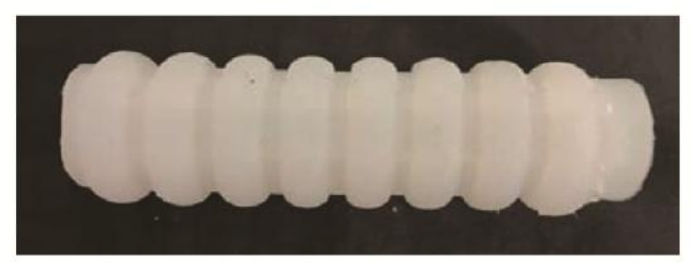

(c) 硅胶纤维复合实物图

图 12 硅胶纤维复合层结构

\section{4 变刚度软体机器人性能测试}

刚度反应了软体机器人抵抗外力的能力, 是重 要评价指标, 为验证所提出机器人的变刚度性能和 稳定性能, 开展了弯曲刚度测试试验和变刚度稳定 性试验。

\section{1 试验平台}

试验平台见图 13a, 包含夹持器、支撑杆、步 进电动机、数字压力传感器和线性导轨。机器人一 端由夹紧装置固定在支架上, 另一端坚直自由悬空, 可方便调整高度和角度。数字压力传感器测量端固 定在线性导轨的滑块上, 导轨由步进电动机驱动, 沿水平方向移动, 其步距角为 $1.8^{\circ}$, 转矩为 $0.9 \mathrm{~N} / \mathrm{m}$, 可实现的控制精度为 $0.1 \mathrm{~mm}$ 。数显压力传感器精度 为 $0.01 \mathrm{~N}$, 量程达 $20 \mathrm{~N}$ 。

测试过程中, 机器人未端与传感器相接触, 传 感器移动的距离由步进电动机控制, 范围为 0 $20 \mathrm{~mm}$, 每次移动 $2 \mathrm{~mm}$, 传感器的读数即为机器人 末端力。

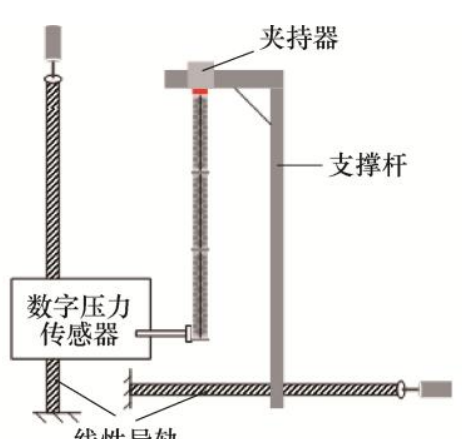

(a) 示意图

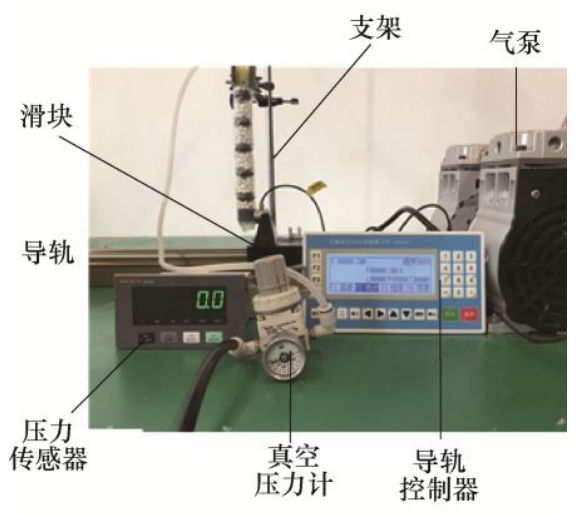

(b) 试验平台

图 13 变刚度软体机器人测试平台

\section{2 刚度测试}

选取半径为 $3 \mathrm{~mm}$ 的填充颗粒, 设置真空压力 分别为 $0 \mathrm{kPa} 、 40 \mathrm{kPa} 、 60 \mathrm{kPa} 、 80 \mathrm{kPa}$, 测量机构 在不同真空压力下末端力与位移的关系, 共进行 4 组试验, 每组重复 4 次, 共计 16 次试验。计算平均 值, 试验结果见图 14a, 可得: 堵塞机构内真空度 越大, 相同位移下传感器测出的末端力越大, 真空 度增量相同时, 末端力的增量逐渐减少。

机器人的刚度 $k$ 表示为

$$
k=F_{\mathrm{c}} / d_{\mathrm{c}}
$$

式中, $F_{\mathrm{c}}$ 末端所受压力, 即传感器测量出的压力; $d_{\mathrm{c}}$ 为机器人末端位移, 即线性导轨滑块位移。真空 压力分别设置为 $0 \mathrm{kPa} 、 20 \mathrm{kPa} 、 40 \mathrm{kPa} 、 60 \mathrm{kPa}$ 、 $70 \mathrm{kPa} 、 80 \mathrm{kPa}$ ，滑块位移范围在 $0 \sim 20 \mathrm{~mm}$ ，每次 移动 $2 \mathrm{~mm}$ 。对每组气压下的试验数据进行拟合, 得 机器人的刚度与输入真空气压的对应关系, 见图 $14 \mathrm{~b}$ 。可得, 随输入真空气压的增加, 机构的刚度逐 渐增加, 且增幅也逐渐增加。

图 $14 \mathrm{c}$ 为机器人的理论刚度仿真值与实际测量 值对比图, 可得, 在真空度 $60 \mathrm{kPa}$ 以下, 理论值和 试验值的差值变化较小, 在 $5.05 \mathrm{~N} \cdot \mathrm{mm}$ 左右, 差值 为实际测量值的 $2.76 \%$ 。当真空度高于 $60 \mathrm{kPa}$, 差 值逐渐增加, 真空度为 $80 \mathrm{kPa}$ 时, 差值达到 
$12.47 \mathrm{~N} \cdot \mathrm{mm}$, 差值为实际测量值的 $5.72 \%$ 。差值逐 渐变大的原因是当真空压力较大时, 机构内颗粒结 构发生变形, 刚度提升趋势减小。

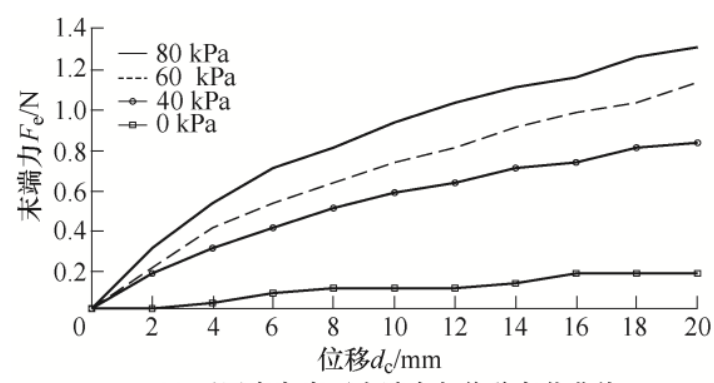

(a) 不同真空度下末端力与位移变化曲线

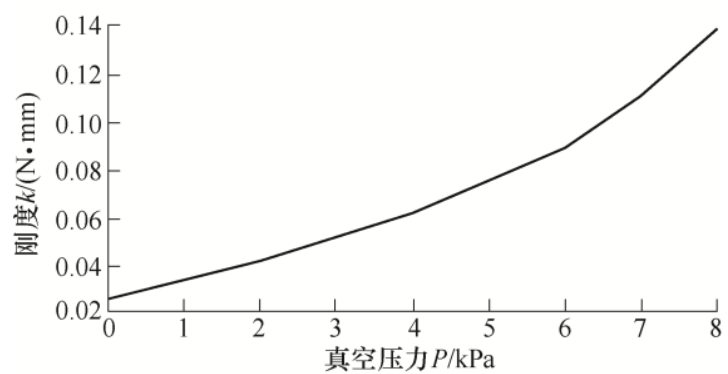

(b) 机构末端刚度与真空压力的关系

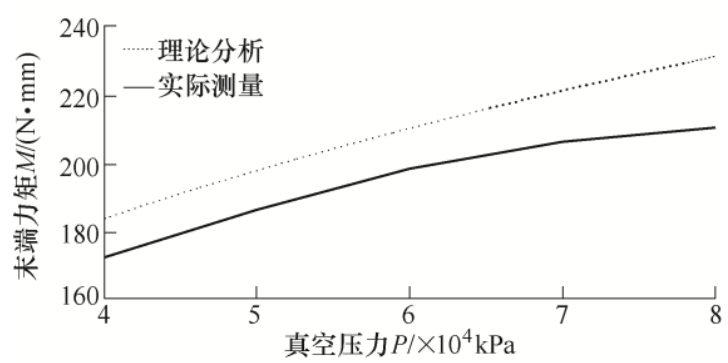

(c) 理论分析和实际测量结果对比

图 14 刚度测试结果

\section{3 影响机器人刚度的因素分析}

第 3 节的理论分析将变刚度机构内填充颗粒视 为整体, 并认为填充颗粒能将真空压力均匀地传递 到接触表面。实际上, 压力的传递与颗粒的数量, 尺寸和结构等均有关, 这些因素都对变刚度机构的 刚度性能产生影响。从颗粒尺寸的角度, 对填充颗 粒半径为 $4 \mathrm{~mm}, 3 \mathrm{~mm}$ 和 $2 \mathrm{~mm}$ 的机构在 $80 \mathrm{kPa}$ 和 $0 \mathrm{kPa}$ 压力下进行测试, 具体数据见图 $15 \mathrm{a}$ 。

对试验数据进行线性拟合，得到不同尺寸颗粒 时机器人的刚度见表 2 。颗粒半径为 $2 \mathrm{~mm}$ 的机构在 $80 \mathrm{kPa}$ 和 $0 \mathrm{kPa}$ 压力下刚度之比为 5.10 , 颗粒半径 为 $3 \mathrm{~mm}$ 的机构在 $80 \mathrm{kPa}$ 和 $0 \mathrm{kPa}$ 压力下刚度之比 为 6.57 , 颗粒半径为 $4 \mathrm{~mm}$ 的机构在 $80 \mathrm{kPa}$ 和 $0 \mathrm{kPa}$ 压力下刚度之比为 4.53 。如果颗粒过小, 根据第 4.4 节对小颗粒与大颗粒堵塞机构的重复定位精度实验 以及 BROWN 教授 ${ }^{[1]}$ 提出的小颗粒机构可知, 小颗 粒机构重复定位精度偏低, 需要改进; 如果颗粒过
大则会使堵塞机构整体结构的偏大化而不利于执行 复杂环境的任务, 以及对驱动的要求过高。因此, 填充颗粒半径为 $3 \mathrm{~mm}$ 的机构刚度变化率最大, 这 对机构优化设计提供了重要参考。

表 2 不同尺寸颗粒机器人刚度结果

\begin{tabular}{cccc}
\hline $\begin{array}{c}\text { 填充颗粒半径 } \\
/ \mathrm{mm}\end{array}$ & $\begin{array}{c}80 \mathrm{kPa} \text { 下刚 } \\
\text { 度 } /(\mathrm{N} / \mathrm{mm})\end{array}$ & $\begin{array}{c}0 \mathrm{kPa} \text { 下刚度 } \\
/(\mathrm{N} / \mathrm{mm})\end{array}$ & 刚度变化之比 \\
\hline 2 & 0.07605 & 0.0149 & 5.10 \\
3 & 0.1355 & 0.0206 & 6.58 \\
4 & 0.1581 & 0.0349 & 4.53 \\
\hline
\end{tabular}

将试验数据处理后, 与仿真数据进行对比见 图 15b, 试验测量的不同尺寸颗粒的刚度效果要低 于理论分析的数值, 且颗粒尺寸越大, 差值越小。

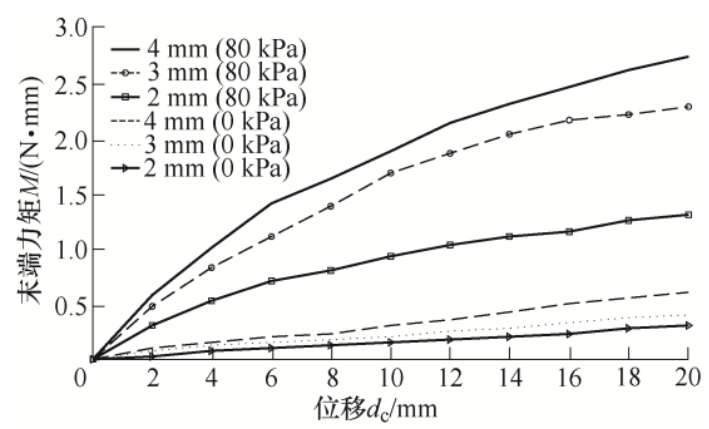

(a) 机器人末端力与位移的关系

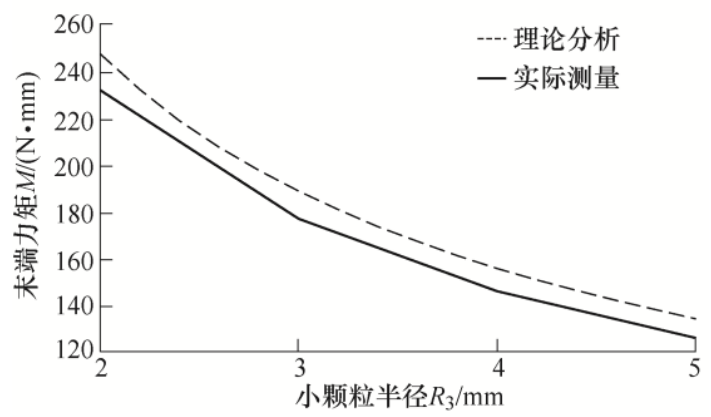

(b) 理论刚度与实验刚度对比

图 15 不同尺寸颗粒对机器人刚度影响分析

选用菱形骨架半径为 $13 \mathrm{~mm}, 14 \mathrm{~mm}$ 和 $15 \mathrm{~mm}$ 的机构进行测试试验, 结果见图 16a。可得骨架半 径越大, 其刚度越大。同时, 随着位移增大, 位移 增量相同时, 末端力的增量在逐渐减小, 这与理论 分析(图 8b)仿真结果相吻合。

根据公式(20), 对末端力和位移的数据进行拟 合可得, 菱形骨架半径为 $13 \mathrm{~mm} 、 14 \mathrm{~mm}$ 和 $15 \mathrm{~mm}$ 时, 机构的刚度分别为 $0.085 \mathrm{~N} / \mathrm{mm} 、 0.133 \mathrm{~N} / \mathrm{mm}$ 和 $0.180 \mathrm{~N} / \mathrm{mm}$ 。将试验数据处理后, 与所得仿真数据 进行对比, 结果见图 16b, 可见, 试验测量出的不 同菱形骨架尺寸的刚度要低于理论分析的结果, 且 骨架尺寸越大，差值越大。 


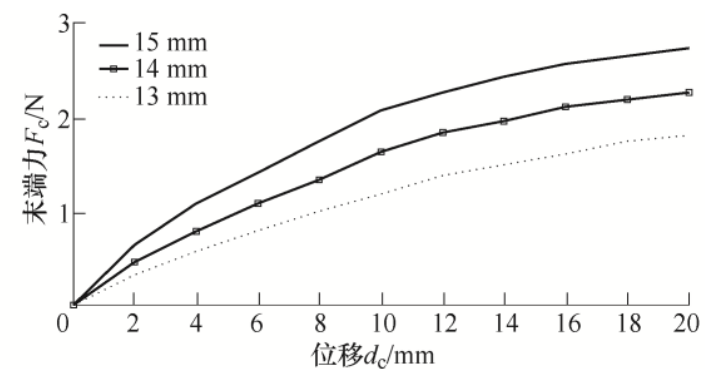

(a) 机器人末端力与位移关系曲线

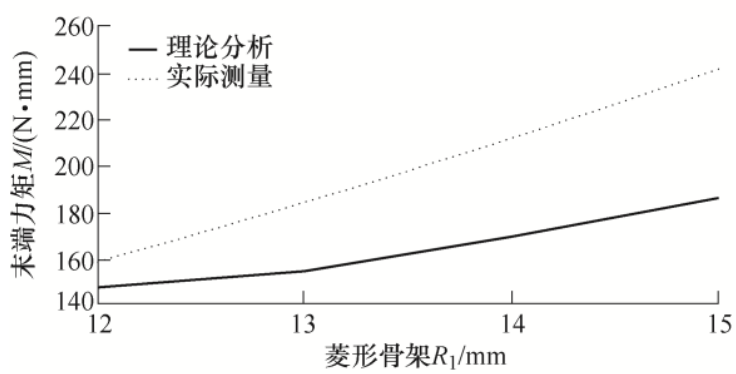

(b) 理论刚度与试验刚度对比

图 16 不同尺寸菱形骨架对刚度的影响

填充颗粒材料分别选取相同半径 $(3 \mathrm{~mm})$ 的刚玉 颗粒、圆黄豆颗粒和聚丙烯颗粒, 在 $80 \mathrm{kPa}$ 的压力 下, 进行测试。结果见图 17, 采用刚玉材质作为填 充颗粒的变刚度结构, 在试验中的刚度表现最好, 这与刚玉颗粒的表面粗粘度直接相关。

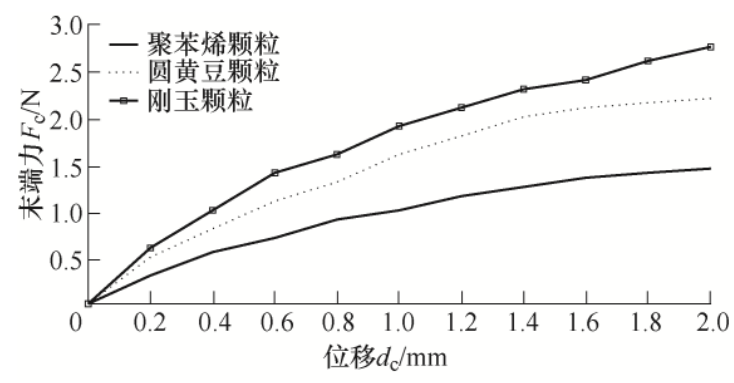

图 17 不同材料填充颗粒的刚度结果

利用公式 (20), 对末端力和位移的数据进行拟合可 得出, 选用聚丙烯材料的机构刚度为 $0.0805 \mathrm{~N} / \mathrm{mm}$, 选用圆黄豆材料的刚度为 $0.131 \mathrm{~N} / \mathrm{mm}$, 选用刚玉材料 的机构的刚度为 $0.158 \mathrm{~N} / \mathrm{mm}$, 可得选用刚玉材料的机 构刚度具有明显优势。

\section{4 重复刚度精度与稳定性测试}

由于软体机器人应用不同尺寸堵塞机构实现变 刚度, 在相同负压驱动下, 即使运动到同一状态, 刚度也会存在差别, 因此, 重复刚度精度是衡量机 器人稳定性的重要参数。应用步进电动机驱动力传 感器每次移动固定距离 $20 \mathrm{~mm}$, 记录传感器数据后, 再还原初始位置, 分别对大球颗粒和小球颗粒堵塞 机构重复测试 100 次。通过 100 组数据计算出的标 准偏差、变异系数和最大绝对偏差, 来衡量机构的
稳定性和刚度精度。

对基于大小颗粒的变刚度机构和基于小颗粒的 变刚度机构进行刚度测试试验后, 对试验所得数据 进行处理, 计算数据的标准偏差, 变异系数和最大 绝对偏差, 结果见表 3 。

由表 2 可知, 在同等试验条件下, 本文提出 的基于大小颗粒组合的机器人的变刚度数据标准 偏差为 $0.0021 \mathrm{~N} / \mathrm{mm}$, 变异系数为 $1.5427 \%$, 而基 于小颗粒的机器人的变刚度数据的标准偏差为 $0.0029 \mathrm{~N} / \mathrm{m}$ !, 变异系数为 $2.1837 \%$, 标准偏差 减小 $27.59 \%$, 变异系数降低 $29.355 \%$, 稳定性明 显提高。本文机构刚度的最大绝对偏差(重复精度) 为 $0.00625 \mathrm{~N} / \mathrm{mm}$, 与小颗粒机构的 $0.0078 \mathrm{~N} / \mathrm{mm}$ 相 比, 降低 $19.8718 \%$, 重复精度明显提高。

表 3 机器人测试刚度数据对比

\begin{tabular}{lccc}
\hline & $\begin{array}{c}\text { 标准偏差 } \\
/(\mathrm{N} / \mathrm{mm})\end{array}$ & 变异系数 $(\%)$ & 最大绝对偏差 $/(\mathrm{N} / \mathrm{mm})$ \\
\hline 本文机构 & 0.0021 & 1.5427 & 0.00625 \\
小颗粒机构 & 0.0029 & 2.1837 & 0.0078 \\
\hline 降低百分比 $(\%)$ & 27.59 & 29.3550 & 19.871795 \\
\hline
\end{tabular}

\section{5 结论}

本文设计了一款包含堵塞变刚度结构层、气动 驱动结构层、硅胶-纤维复合结构层三层结构的气动 与堵塞机构耦合的变刚度软体驱动器; 建立了变刚 度软体机器人的刚度模型, 推导出机构末端的刚度 与真空压力、大球尺寸、菱形骨架尺寸和填充颗粒 参数之间的关系; 制作了变刚度软体机器人样机, 进行刚度和弯曲性能测试试验, 利用多次试验所得 数据的标准偏差, 变异系数和平均绝对误差表征了 机器人的变刚度特性和刚度稳定性能。

仿真和试验表明: 应用半径为 $2 \mathrm{~mm}, 3 \mathrm{~mm}$ 和 $4 \mathrm{~mm}$ 的刚玉颗粒, 在 $-80 \mathrm{kPa}$ 真空压力下刚度变化 比分别为 $5.10,6.58$ 和 4.53 。因此, 填充颗粒半径 为 $3 \mathrm{~mm}$ 的机构刚度变化率最大。所提出的基于大 小颗粒组合的机器人变刚度的标准偏差为 $0.0021 \mathrm{~N} / \mathrm{mm}$, 变异系数为 $1.5427 \%$, 而基于小颗 粒的机器人的变刚度数据的标准偏差为 $0.0029 \mathrm{~N} / \mathrm{mm}$, 变异系数为 $2.1837 \%$, 标准偏差减 小 $27.59 \%$, 变异系数降低 $29.355 \%$, 稳定性明显提 高。本文机构刚度的最大绝对偏差 (重复精度) 为 $0.00625 \mathrm{~N} / \mathrm{mm}$, 与小颗粒机构的 $0.0078 \mathrm{~N} / \mathrm{mm}$ 相 比, 降低 $19.8718 \%$, 重复精度有所提高。 


\section{参 考 文 献}

[1] AMEND J R, BROWN Jr E, RODENBERG N, et al. A positive pressure universal gripper based on the jamming of granular material[J]. IEEE Trans. Robotics, 2012, 28(2): 341-350.

[2] HOU Taogang, YANG Xingbang. Design and experiment of a universal two-fingered hand with soft fingertips based on jamming effect[J]. Mechanism and Machine Theory, 2019, 133: 706-719.

[3] ZHU Mingzhu. A fully multi-material three-dimensional printed soft gripper with variable stiffness for robust grasping[J]. Soft Robotics, 2019, 6(4): 1-13.

[4] LI Yingtian, CHEN Yonghua, LI Yunquan. Distributed design of passive particle jamming based soft grippers[C]// 2018 IEEE International Conference on Soft Robotics (RoboSoft), April 24-28, 2018, Livorno, Italy, Malaysia: IEEE, 2018: 547-552.

[5] LI Yingtian, CHEN Yonghua, YANG Yang. Soft robotic grippers based on particle transmission[J]. IEEE/ASME Transactions on Mechatronics, 2019, 24(3): 969-978.

[6] AMEND J, CHENG N, FAKHOURI S, et al. Soft robotics commercialization : Jamming grippers from research to product[J]. Soft Robotics, 2016, 3(4) : 213-222.

[7] WEI Ying, CHEN Yonghua, YANG Yang, et al. A soft robotic spine with tunable stiffness based on integrated ball joint and particle jamming[J]. Mechatronics, 2016, 33: 84-92.

[8] WU Zhaoping, LI Xiaoning, GUO Zhonghua. A novel pneumatic soft gripper with a jointed endoskeleton structure[J]. Chinese Journal of Mechanical Engineering, 2019, 32(1): 78-89.

[9] WEI Dunwen, GAO Tao, MO Xiaojuan, et al. Flexible bio-tensegrity manipulator with multi-degree of freedom and variable structure[J]. Chinese Journal of Mechanical Engineering, 2020, 30(3): 56-66.

[10] KIM Y J, CHENG S, KIM S, et al. A novel layer jamming mechanism with tunable stiffness capability for minimally invasive surgery[J]. IEEE Trans. Robot, 2013, 29(4): 1031-1042.

[11] CHENG N G, LOBOVSKY M B, KEATING S J, et al. Design and analysis of a robust, low-cost, highly articulated manipulator enabled by jamming of granular media[C]// 2012 IEEE International Conference on
Robotics Automation, May 14-18, 2012, RiverCentre, Saint Paul, Minnesota, USA: IEEE, 2012: 4328-4333.

[12] LIN Nan. IMU-based active safe control of a variable stiffness soft actuator[J]. IEEE Robotics and Automation Letters, 2019, 4(2): 1247-1254.

[13] 张明, 房立金, 孙风, 等. 永磁变刚度柔性关节的力 学分析与控制器设计 $[J]$. 机械工程学报, 2019, 55(5): 89-96.

ZHANG Ming, FANG Lijin, SUN Feng, et al. Mechanical analysis and controller design of the permanent magnetic variable stiffness flexible joint[J]. Journal of Mechanical Engineering, 2019， 55(5): 89-96.

[14] WALL V, DEIMEL R, BROCK O. Selective stiffening of soft actuators based on jamming[C]// 2015 IEEE International Conference on Robotics Automation, May 26-30, 2015, Seattle, Washington, USA: IEEE 2015, 252-257.

[15] WEI Ying, CHEN Yonghua, REN Tao, et al. A novel, variable stiffness robotic gripper based on integrated soft actuating and particle jamming[J]. Soft Robotics, 2016, 3(3): 134-143.

[16] STELTZ E, MOZEIKA A, RODENBERG $\mathrm{N}$, et al. JSEL : Jamming skin enabled locomotion[C]// 2009 IEEE/RSJ International Conference on Intelligent Robots and Systems, October 11-15, 2009, St.Louis, USA: IEEE 2009: 5672-5677.

[17] JIANG Pei. A variable stiffness gripper based on differential drive particle jamming[J]. Bioinspiration \& Biomimetics, 2019, 14(5): 1-14.

[18] WANG Tao, ZHANG Jinhua, LI Yue, et al. Electrostatic layer jamming variable stiffness for soft robotics[J]. IEEE/ASME Transactions on Mechatronics, 2019, 24(2): 424-433.

[19] 姚立纲, 李敬仪, 东辉. 气动软体机械臂模块变刚度性 能分析 $[J]$. 机械工程学报，2020，56(9)：36-44.

YAO Ligang, LI Jingyi, DONG Hui. Variable stiffness analysis on a pneumatic soft manipulator[J]. Journal of Mechanical Engineering, 2020， 56(9): 36-44.

[20] 王田苗, 郝雨飞, 杨兴帮, 等. 软体机器人: 结构、驱 动、传感与控制 $[J]$. 机械工程学报, 2017, 53(13): 1-13. WANG Tianmiao, HAO Yufei, YANG Xingbang, et al. Soft robotics: Structure, actuation, sensing and control[J]. Journal of Mechanical Engineering, 2017, 53(13): 1-13.

[21] ANDREW D, MARCHESE, ROBERT K, et al. A recipe for soft fluidic elastomer robots[J]. Soft Robotics, 2014, 
2(1): 7-25.

[22] MARCHESE A, TEDRAKE R, RUS D. Dynamics and trajectory optimization for a soft spatial fluidic elastomer manipulator[J]. International Journal of Robotics Research, 2016, 35(8): 1000-1019.

[23] MARCHESE A, RUS D. Design, kinematics, and control of a soft spatial fluidic elastomer manipulator[J]. International Journal of Robotics Research, 2016, 35(7):
840-869.

[24] GENT A N. On the relation between indentation hardness and Young's modulus[J]. Rubber Chemistry and Technology, 2008, 31(4): 896-906.

作者简介: 徐丰羽, 男, 1979年出生, 博士, 教授, 硕士研究生导师。 主要研究方向为机器人及自动化, 机电一体化技术。

E-mail: xufengyu598@163.com 